\title{
The origin and functions of adjunctive behavior
}

\author{
JOHN L. FALK \\ Rutgers University, New Brunswick, New Jersey 08903
}

\begin{abstract}
The major determinants of schedule-induced or adjunctive behavior are reviewed briefly. Adjunctive behavior and its ethological equivalent, displacement activity, has a stabilizing function on agonistic, mating, parental, and intermittent-feeding behavior when any of these activities are in unstable equilibrium with an escape vector. This buffering action of adjunctive behavior is analogous to the diversity-stability rule of ecology in which an increase in the diversity of species stabilizes the populations of the component species, thereby preventing extinction. Opposing behavioral vectors in unstable equilibrium can function to exaggerate certain behavioral adjuncts that preexist in a situation. The resulting increase in diversification (information) augments the overall stability of the opposing-vector circumstance, conserving the context. This strengthening process is discussed in relation to ritualization and the preadaptation of exaggerated behavior to new functions.
\end{abstract}

The nature and determinants of adjunctive behavior were described in a previous article (Falk, 1971). Briefly, "adjunctive behavior" refers to behavior that is maintained at a high probability by stimuli which derive their exaggerated reinforcing efficacy primarily as a function of schedule parameters governing the availability of another class of reinforcing events. The present account will attempt to answer the questions of how the adjunctive determination of behavior may have evolved and what adaptive functions it might serve. Considerable information has accumulated concerning the variables which determine adjunctive behavior. A few of the major features will be described at the outset, but the treatment is far from complete. This is neither a review nor even a synoptic summary.

Animals (rats, mice, pigeons, squirrel and rhesus monkeys, chimpanzees) reduced in body weight and given small portions of food at certain fixed or irregular times in daily sessions quickly develop excessive and persistent behavioral adjuncts. For example, rats earning $45-\mathrm{mg}$ food pellets by leverpressing on a variable-interval 1-min schedule or on a fixed-interval $90-\mathrm{sec}$ schedule for a few hours per day drink extensive draughts of water after consuming each pellet (Falk, 1961, 1969). This scheduleinduced polydipsia continues daily as long as the generator schedule conditions remain in effect. Approximately 10 times the amount of water is drunk compared to a condition in which the animal is given the same amount of food daily as a single portion and remains in the situation for the same

This manuscript was facilitated by support from Grant DA 1110 from the National Institute on Drug Abuse, Grant AA00253 from the National Institute of Alcohol Abuse and Alcoholism, and a Biological Sciences support grant (NIH) to Rutgers University. The author's address is: Psychology Building, Rutgers University, Busch Campus, New Brunswick, New Jersey 08903. number of hours. The animal is never water deprived, yet the intermittent delivery of food increases the reinforcing value of water by a mechanism which is not at all explicable by metabolic or water balance considerations (Falk, 1969).

The generator conditions produced by intermittent food schedules can induce exaggerated behaviors other than polydipsia. Schedule-induced attack against a conspecific, an inanimate object, a conspecific model, or a conspecific visual photo target have been studied extensively (e.g., Azrin, Hutchinson, \& Hake, 1966; Cohen \& Looney, 1973; Flory, 1969; Hutchinson, Azrin, \& Hunt, 1968). Scheduled food delivery also can induce escape behavior. Animals will emit operant behavior which results in the termination of the schedule (time out) or in the removal of stimuli associated with the schedule (escape from the discriminative stimuli) (e.g., Azrin, 1961; Brown \& Flory, 1972; Thompson, 1964). Schedule-induced adjunctive wheel running (Levitsky \& Collier, 1968), pica (Villarreal, Note 1), pecking (Miller \& Gollub, 1974), and airstream licking (Mendelson \& Chillag, 1970) are other activities which occur excessively and in a fixed temporal relation relative to the inducing events (food-portion delivery) of the generator schedule. However, generator conditions do not necessarily involve food deprivation and the intermittent delivery of small food portions. Recent research with rats (King, 1974; Wayner, Singer, Cimino, Stein, \& Dwoskin, 1975) and humans (Kachanoff, Leveille, McLelland, \& Wayner, 1973; Wallace, Singer, Wayner, \& Cook, 1975; Wallace \& Singer, 1976) has demonstrated the induction of various adjunctive behaviors in which the generator conditions constituted the scheduling of water, running-wheel access, monetary gain, game playing, or problem solving rather than food. Thus, adjunctive behavior reveals a considerable degree of 
generality both in the kinds of commodities or behavior sequences whose intermittency constitutes a generator condition and in the variety of resulting adjunctive activities.

While a host of conditions are demonstrable modulators of adjunctive behavior, two determinants are of particular importance: the intermittence value of the generator schedule and the deprivation status of the scheduled consummatory behavior. When the first of these factors, intermittence value, was varied systematically, a somewhat counterintuitive function was obtained. Many fixed-interval schedule values between 2 and $300 \mathrm{sec}$ were explored for the rat. Adjunctive drinking increased as a function of the fixed-interval food schedule value up to 120-180 sec and decreased to a low level at the 300 -sec value (Falk, 1966). Subsequent experiments have confirmed this bitonic function, not only for schedule-induced polydipsia in the rat (e.g., Flory, 1971), but also for other adjunctive activities and for species other than the rat. For example, a bitonic function has been reported for schedule-induced polydipsia in the rhesus monkey (Allen \& Kenshalo, 1976) and schedule-induced attack and escape in the pigeon (Brown \& Flory, 1972; Flory, 1969).

The other major factor determining the rate of adjunctive behavior, the deprivation status of the scheduled consummatory behavior, has received less experimental attention than the bitonic function, but the results have been quite consistent. The degree of schedule-induced polydipsia (Falk, 1969), attack (Dove, 1976), and airstream licking (Chillag \& Mendelson, 1971) produced by intermittent food schedules were all inverse functions of the bodyweight level at which animals were maintained as determined by the total food rations allowed.

Thus, adjunctive activities are systematically related to two main constraining conditions. The activities are bitonic functions of the generator intermittence value and increasing monotonic functions of the degree of deprivation. They are excessive and persistent. A behavioral phenomenon which encompasses many kinds of activities and is widespread over species and high in predictability ordinarily can be presumed to be a basic mechanism contributing to adaptation and survival. The puzzle of adjunctive behavior is that, while fulfilling the above criteria, its adaptive significance has escaped analysis. Indeed, adjunctive activities have appeared not only curiously exaggerated and persistent, but also energetically quite costly. They do not have the relation of unconditioned, conditioned or operant responses, or fixed-action patterns to the conditions generating them. Consequently, traditional accounts of behavior afforded by Pavlovian and operant conditioning and by ethology seem not to apply. However, similarities between the conditions producing both adjunctive and displacement activities have been suggested (Falk, 1971), and this analysis will be expanded.

The reader is now requested to bear with me while the case for certain ecological and evolutionary analogies is slowly, and I hope not too painfully, built. These will converge later in the paper to provide a basis for ascertaining the origin and functions of the seemingly profligate behaviors termed "adjunctive."

\section{THE ECOLOGICAL ANALOGY: STABILIZING FUNCTION OF NETWORKS}

An ecosystem is defined as all the organisms within a given region, their interactions with the physical environment, and, most importantly, their interactions among themselves. These latter interactions constitute the food web or predator-prey relations. The food web consists of food chains, with each link in the chain being a particular predator-prey relation. The links are located at different trophic levels. The green plants, dependent upon energy from the sun, define the first level, the herbivores consuming them define the second level, and the carnivores living on them compose the third level. Carnivores consuming third-level carnivores would constitute a fourth trophic level. Feeding may span more than one trophic level. For example, the omnivores eat both plant and animal materials.

The food webs of the species populations constituting different ecosystems can take many different forms. Nevertheless, a roughly acceptable notion, at least as a point of departure, is that the stability of an ecosystem increases as the number of food web linkages increases. Thus, the greater the number of plant and animal species participating in an ecosystem, the less each species will fluctuate in numbers. The argument that species community stability is dependent upon trophic web complexity stems from several lines of evidence. Both mathematical considerations of predator-prey interactions (MacArthur, 1955) and field observations indicate that a community containing just a single prey species and its enemy oscillates widely in numbers (Elton, 1958, p. 146; Odum, 1971, chap. 7). Although there is little stability, extinction does not necessarily occur. The community is subject to periodic "outbreaks" of each species, especially in simple environments (e.g., small islands, cultivated monocultures, arctic regions). The addition of one or more prey species would diminish such fluctuations. The ease with which invading species are able to colonize the relatively simple environments of islands containing rather few species further illustrates the vulnerability of simple ecosystems. The harsh conditions of the arctic apparently limit the number of species, and stability conditions are correspondingly delicate (MacArthur, 1955). Such regions are, as 
would be expected, characterized by large, cyclic oscillations in their fauna which "may be due in part to the communities not being sufficiently complex to damp out oscillations" (Hutchinson, 1959). By contrast, the tropical rain forest with a high degree of trophic complexity is unusually stable (Elton, 1958, pp. 148-149).

As sparse islands are susceptible to species invasions and the arctic to population oscillations of large magnitude, the artificially simplified crop communities planted in large stands by man are also extremely vulnerable. Monoculture reduces the diversity of flora, and such special crops usually lack the development of typical attached fauna. Generalized pesticides further reduce both herbivore and carnivore species diversities, sometimes quite drastically, thereby limiting pest enemies along with the pests. Thus, pest outbreaks are frequent due to the extreme simplicity of the monoculture. Also, pest enemies fail to proliferate as these parasites and predators often require nourishment derived from the secretions of plants absent from monocultures. In contrast with this picture, pest outbreaks do not occur in tropical forests where the great diversity of plant and animal species constitutes a highly complex and stable community. Likewise, the intercropping of a great diversity of plants practiced by the swidden agriculturalists of Malaysia and Indonesia imitates the diversity and stability of the jungle which it temporarily replaces (Geertz, 1963, chap. 2).

Community stability, then, is a function of the number of links among species in the food web, or stated another way, "the amount of choice of the energy in going through the web measures the stability" (MacArthur, 1955). This can be viewed in terms of what occurs when one species increases or decreases. As it increases, competitors on the same trophic level may decrease, and predators and parasites may switch to the more abundant prey. Conversely, a decrease in the species would produce a switch in preference by predators to less rare, alternate prey. The more diverse and complex ecosystems may be more stable since the individual species populations would be protected from the possibility of large fluctuations by the buffering action of other species groups. This relation is sometimes referred to as the diversity-stability rule (Wilson \& Bossert, 1971, p. 140). The rule was formalized by MacArthur (1955), who applied the ShannonWiener formula for the measurement of information in a system to the analysis of ecological communities. The diversity or stability of a community is

$$
H=-\sum_{i}^{s} p_{i} \log p_{i},
$$

where $s$ is the number of species considered and $p_{i}$ is the proportion which the population of the $i^{\text {th }}$ species is of the total number of individuals composing the species population.

The rule and its most frequent mathematical expression do not have the status of a mathematical theorem. Mathematical models have been proposed that do substantiate the rule given certain assumptions or conditions (Leigh, 1965; May, 1973, pp. 102107; Webber, 1974). But, in general, "the greater the size and connectance of a web, the larger the number of characteristic modes of oscillation it possesses: since in general each mode is as likely to be unstable as to be stable (unless the increased complexity is of a highly special kind), the addition of more and more modes simply increases the chance for the total web to be unstable" (May, 1973, p. 75). However, May (1973, pp. 75-76) indicates that "the balance of evidence would seem to suggest that, in the real world, increased complexity is usually associated with greater stability." This occurs because ecosystems are mathematically atypical. "Nature represents a small and special part of parameter space" (p. 76). A similar conclusion was reached by Rogers and Hubbard (1974) in examining a parasite-host model in the light of actual data on insect parasites and predators. The real species populations did not show the instability predicted by the deductive model. On the other hand, there are data which indicate that an increase in species diversity does not always yield an increase in stability (Hairston et al., 1968; Watt, 1968, pp. 43-45). Thus, the diversity-stability rule must be treated with caution. Its deductive underpinnings are suggestive, but not compelling. The relevant data likewise fall short of being entirely persuasive. Nevertheless, the rule is in accord with the analysis of several genera! ecological situations, as described at the beginning of this section. It is worth considering its applicability to analogous relations in the realm of behavior.

Just as an uncomplicated ecosystem is subject to instability or wide, cyclic oscillations which may be remedied by an information increase in the species network, an increase in the probability of preexisting behaviors (viz, adjunctive behavior generation) may limit unstable fluctuations in situations containing relatively few behavioral vectors. Thus, the augmented adjunctive activity in a behaviorally unstable situation may serve to strengthen a fluctuating or deteriorating behavior rather than to occlude it. On the other hand, the vulnerability of a relatively simplified island or monoculture ecosystem to invasion by a colonizing species has its counterpart in the excessive and intrusive aspects of adjunctive behavior in the relatively barren situations that constitute both controlled experimental spaces and life situations lacking alternative opportunities or repertoires. Thus, adjunctive behavior can either stabilize preexisting, adaptive behavior in an otherwise unstable situation or be so invasive in vulnerable situa- 
tions that it eliminates other species of behavior. Whether this latter outcome is creative or toxic for the organism depends upon the overall adjustive value of the newly dominant behavior (Falk, 1971). If the adjunctive behavior is chronic alcohol or other drug overindulgence, then it would be considered behaviorally disadvantageous (Falk \& Samson, 1975). If it is a persistent artistic or scientific endeavor, it would be judged as creatively adaptive. The exaggerated and persistent aspect of adjunctive behavior occurring in vulnerable contexts should not be overemphasized. Rather, the conservative, stabilizing function of adjunctive behavior has probably provided its major, selective, evolutionary advantage.

\section{ROMER'S RULE AND PREADAPTATION}

Evolutionary change often is described in terms of special innovations which meet the specific challenges and opportunities posed by altered environments. While new circumstances may require new adaptive modes, there is also a conservative side to evolutionary change. The variant selected may be one which initially facilitates the continuation of a previous adaptive mode in altered circumstances. Only later does the selective change develop a truly innovative relation to the altered ecological niche of the species. For example, Romer (1959) describes the evolution of the amphibians from lungfish living in stagnant fresh waters. With the seasonal droughts of the Devonian period, the pools would have shrunk and become crowded as well as exhausted of food. Such periodic recessions of their pools produced a selection pressure favoring lungfish with fins strong enough to get them to another pool. Strong fins which could support weight out of the water were developed initially not to invade the land, but rather to continue an aquatic life by moving to new pools. "Land limbs were developed to reach the water, not to leave it" (Romer, 1959, p. 94). But once this rather conservative step toward maintaining their aquatic existence had been taken, it permitted the exploitation of a new ecological niche and a major evolutionary opportunity. The innovation of terrestrial life was actually a consequence of selection pressure to maintain an aquatic one. In a similar vein, Romer $(1959$, p. 327$)$ suggests that ancestral man may have been forced into ground locomotion from his arboreal existence because the expanding grasslands and the consequent shrinking of forest areas required him to cross grassy prairies to reach patches of forest. Just as walking developed so that amphibians could reach pools, walking in man's ancestors may have developed so that he could maintain an arboreal life.

These examples have been generalized by Hockett and Ascher (1964) into what they call Romer's rule: "The initial survival value of a favorable innovation is conservative, in that it renders possible the maintenance of a traditional way of life in the face of changed circumstances." Later, such innovations may have more radical evolutionary implications, such as a changeover from aquatic or arboreal to terrestrial life. But, according to Romer's rule, the immediate consequence of successful innovation is a stabilization of the current adjustive context. The key notion here is that a contextual conservatism, operating through innovation, results in the stabilization of an unstable situation. In Romer's examples, the innovations were in mobility modes, but there are other ways in which innovations can occur. Rather than developing a new mobility mode, a species may extend greatly a particular mode by gradually coming under the guidance of new controlling stimuli. Skinner (1975) has described how, as the continents drifted slowly apart, distant migrations to breeding grounds in turtles, fish, eels, and birds could have developed gradually from the selection of genotypes and the transfer of stimulus control. Rather than new modes of mobility, various migrating species have deployed increased responsiveness to new classes of stimuli which have come to control the swimming or flying and permit the extensive trips necessary to retain their traditional breeding grounds.

Romer's rule can be applied, thus, not only to innovation in the modes of mobility, but to the extreme extensions of mobility classed as migrations. Since the rule deals with contextual conservatism, it is even more probable that mechanisms would evolve which limit mobility in situations where this maintains currently favorable adjustments. One way limited mobility occurs is by ancillary behavior coming under the strong control of stimuli in the immediate environment. It was indicated in the previous section that such local diversification of behavior might promote the stabilization of an unstable situation. How this occurs will be amplified in later sections.

The contextual conservatism (Romer's rule) of selective modifications which occur in response to a changed environment does not preclude the opportunistic, adaptive significance these innovations may possess with respect to other niche possibilities. The conditions that allow some structure or function to change from control by one kind of selective advantage to another have been described by evolution theorists as preadaptation. Bock (1959) considers a structure "to be preadapted for a new function if its present form which enables it to discharge its original function also enables it to assume the new function whenever need for this function arises." The structure is molded into its preadapted form by the selective advantages it affords the original func- 
tion. For example, vertebrate cutaneous vascularization is well developed. It has a respiratory function in fish and amphibians, serves to take in or discharge heat in reptiles, and maintains body temperature within narrow limits in warm-blooded animals (Cowles, 1958). The basic structure (cutaneous vascularization), while selected originally for its respiratory function, was preadapted for temperature regulation. Another example is afforded by the remarkable degree to which the reptiles ancestral to birds were preadapted for flight. These reptiles were arboreal, bipedal, with strong anterior extremities; even feathers were present (Mayr, 1960).

Before applying Romer's rule and the notion of preadaptation to the origin and functional implications of adjunctive behavior, the nature of displacement activity will be discussed, as it forms a bridge between phenomena observed in natural settings and the adjunctive behavior generated in operant conditioning situations.

\section{DISPLACEMENT ACTIVITIES: STIMULUS CONTROL AND FUNCTIONAL SIGNIFICANCE}

The theory of natural selection implies that the behavior patterns characterizing different animal species are interpretable in terms of their adaptive values. Displacement activities are difficult to interpret within this framework, as the behavior sequences are curiously intrusive. They often are described as "out-of-context" behaviors, not only in relation to the behaviors occurring immediately before and after the displacement activity, but also as adjustments to the current environmental situation. These "incongruous" and "irrelevant" activities were related to Freudian symptomatic actions in early ethological theory and viewed as "outlets through which the thwarted drives can express themselves in motion" (Tinbergen, 1952). The notion that displacement behavior functions to rid the organism of surplus and potentially damaging impulses was abandoned later by Tinbergen (1964) and other ethologists for various reasons. Chiefly, it became increasingly evident that the activities were not so irrelevant to the current stimulating circumstances as had been supposed.

Displacement activities occur in agonistic, sexual, or parental situations and specifically in conjunction with interruptions in the progress of behaviors characteristic of these situations. Southern cormorants may show displacement brooding in intervals of fighting, or may displacement-bathe when surprised by a human intruder (Armstrong, 1965 , p. 109). Many species of birds show displacement feeding and preening activities during intervals in intraspecific fighting or mating (Tinbergen, 1952).
In experiments where one or more eggs of the blackheaded gull were removed from the nest, vigorous displacemeni preening or nest building occurred as reactions to the interferences with brooding (Moynihan, 1953). Displacement nest digging and fanning are observed in the male three-spined stickleback during courtship behavior when the female fails to follow the male toward the nest (Tinbergen \& VanIersel, 1947).

In general, a wide range of displacement activities has been studied in various species, including mammalian forms. Displacement feeding, drinking, sexual display, mounting, sleeping, and grooming have been observed, as well as those activities mentioned previously. The situations producing displacement activities involve interference with appetitive or consummatory behavior which is in progress. This can occur in several ways (Armstrong, 1950; Tinbergen, 1952). Another organism or physical event may intrude upon the situation. Incompatible behaviors can alternate and inhibit each other. This second type of event is described in ethological accounts as a conflict situation either between sexual and agonistic behaviors in courtship, or between agonistic and incubation behaviors in a brooding situation. The conditions under which conflicting behaviors occur also give rise to various displacement activities. Finally, a behavior can be terminated rapidly by the sudden cessation of stimuli eliciting or sustaining the behavior. For example, "the rednecked phalarope, where the female takes the initiative in courtship and coition ... of ten attacks the male immediately after coition. Also, she goes on land and indulges in a bout of nest-building", (Tinbergen, 1952). In a similar vein, Armstrong (1950) notes that displacement activities occur "after a goal has been attained or the threshold of expression of a behavior-pattern has been raised." It is important to note that, in all of the above situations, the ongoing behavior was interfered with or summarily terminated and that displacement activities resulted. These curious outcomes are no longer looked upon as an epiphenomenon of central nervous design ("sparking-over"). It is recognized now that whatever may be the ultimate explanation of their causation, the dynamics of displacement activities are under the control of current environmental stimuli.

Stimulus control factors are important in determining the particular displacement activities produced. For example, Morris (1954) noted that the displacements of the zebra finch depended upon what stimuli were nearby. Feeding was the displacement behavior observed when food was in the vicinity, mounting occurred when a female was near, and when neither were easily available, sleep or comfort responses resulted. Formerly, it had been 
assumed that the stimulus situation appropriate to the observed displacement activities was absent, and that the behaviors were thus entirely incongruous. But increasing evidence pointed to the conclusion that when the behavior dominating a situation becomes, for whatever reason, impeded, behavior appropriate to other concurrent stimuli becomes primary (Vanlersel \& Bol, 1958). Events which impede the behavior dominating the situation, whether these events are barriers, intrusions by a competing conspecific or potential predator, conflicting behavior, or the absence of appropriate sustaining stimuli, all produce displacement activities by a permissive action. Detailed analyses of displacement fanning in the three-spined stickleback (Sevenster, 1961) and displacement preening in the chaffinch (Rowell, 1961) showed that external stimuli which facilitated the normal performance of these activities also increased their probability when they occurred as displacement behaviors. Experiments by McFarland (1965) on displacement feeding in the water-deprived Barbary dove induced in a variety of carefully controlled situations demonstrated the importance of both behavioral history and current situational stimuli.

As indicated previously (Falk, 1971), there is continuity in the processes determining displacement and adjunctive behaviors. Both occur when powerfully determined, ongoing behavior is impeded; in both, the particular behavior next in evidence is a function of available facilitating stimuli. Further, the behaviors in both situations have a certain aspect of incongruity: they are excessive and appear to contribute little of adaptive significance to the behavioral situation impeded.

It is worth considering this last point-the intrusive and incongruous aspect of displacement and adjunctive behaviors. As noted, displacement behaviors are under the control of facilitating stimuli and thus not entirely incongruous with respect to situational vectors. Likewise, adjunctive behaviors are not newly engendered activities. They are increases in the magnitude and probability of behaviors already present as moderate, base-rate responses to the general situation (Falk, 1971). Both displacement and adjunctive activities probably are produced by much the same stimuli that produce these behaviors (drinking, aggression, nest-building, etc.) in other, more usual situations. The unusual features involve the temporal locus of such behavior and its apparently nonfunctional exaggeration.

Persistent, nonfunctional behaviors with wide species representation would be something of a puzzle to explain in terms of natural selection. But recent work on displacement indicates that the process may possess certain adaptive functions. Wilz (1970) studied nest-related displacement activities (fanning, creeping through) which occur during courtship in the male three-spined stickleback. These displacement activities are quite frequent following "dorsal pricking," in which, instead of leading the female towards the nest, the male pushes the female away with the dorsal side towards the water surface. Such pricking occurs if the male has just been stimulated by the intrusion of another male. Experimental analysis revealed that the displacement activities facilitated the transition from aggression to courtship. When nest creepingthrough responses were prevented after pricking, biting of the female was frequent and zig-zag leading was infrequent. The displacement activities were a cause rather than an effect of a transition from aggressive to sexual responses. The ritualization of displacement activities into communicative displays which function to mitigate aggression and promote sexual responses in partners has been well described (Tinbergen, 1952). It now appears that displacement activities may be effective in promoting this transition in the performer prior to their selective ritualization into communicative displays.

There are numerous descriptions which suggest that displacement activities function directly as distractions luring predators away from the bird's nesting area. Armstrong (1949a) notes that displacement brooding in a spot where there are neither eggs nor chicks leads predators away from the actual nest site. Displacement feeding in the lapwing occurs when it does not approach its nest in the presence of a predator. The feeding behavior may function to deceive predators in that they are less likely to stalk a bird apparently feeding than one brooding or tending chicks (Armstrong, 1949a). More complex diversionary displays, such as injury simulation, arise through the ritualization of agonistic and mating display components (Armstrong, 1949b; Williamson, 1950).

These studies indicate that, even prior to their ritualization into display, displacement behaviors afford the organism definite adaptive advantages. Armstrong (1950) suggests how various maintenance activities such as nest building and nest lining have evolved directly from displacement movements. Species which are prone to displacement activities, according to his analysis, exhibit these modified behaviors when faced with altered environmental circumstances. This enables them to incorporate these modifications into new adaptive patterns rather than requiring the evolutionary construction of adjustive modifications more slowly from smaller behavioral units. Alcock (1972), in a similar vein, has shown how various instances of the use of tools by feeding animals could have arisen directly from displacement and redirection activities. For example, Darwin's woodpecker finch of the Galapagos carries 
about a cactus thorn or twig with which it pokes into tree cracks, dislodging insects and larvae which it then seizes (Lack, 1947, pp. 58-59). This finch has a beak and tongue length not as suitable as a true woodpecker's for retrieving its prey from crevices. Alcock (1972) suggests that seeing nearby prey obtainable only intermittently may have produced nest-material-gathering displacement activity with nearby twigs. If reattracted by an insect while still holding a twig, a jab of the beak could now be effective in dislodging the prey. Selection pressure would then favor displacement-prone members of the species.

\section{RITUALIZATION, ROMER'S RULE, AND THE ADAPTIVE VALUE OF ADJUNCTIVE BEHAVIOR}

Ritualization, a concept introduced into ethology by Huxley, is defined as the process whereby maintenance and displacement activities have become specialized for communicative functions through selective pressures. The resulting displays are thought to provide unambiguous signals which minimize damage in intraspecies agonistic encounters and promote efficient releasing stimuli in sexual and social bonding situations (Huxley, 1966). However, the agonistic encounters involve not only intraspecies signals but also interspecies predator-prey relations. The latter include ritualized activities resulting in injury- and chick-simulation distraction displays (Armstrong, 1949a, 1949b; Williamson, 1950). Intimidation and cryptic displays occur in moths. Wing "eyespots" are revealed in the intimidation display which mimic predator (owl) eye patterns, and unresponsiveness to strong stimulation (e.g., handling) by potential predators characterizes the cryptic display (Blest, 1957). Distraction, intimidation, and cryptic displays having interspecies predator-defense value appear to be products of ritualization processes similar to those yielding intraspecies communicative functions.

As a behavior becomes ritualized, it is thought to be released by stimulus situations different from those that produced the ancestral behavior. For example, the preening involved in courtship display is described as being emancipated not only from its original maintenance and comfort context but also from a displacement role. It is evoked by a stimulus complex relevant to courtship and mating. As ritualization proceeds, presumably the behavior loses its responsiveness to the usual stimuli that facilitate or inhibit it in other contexts, and in this sense it progresses beyond displacement behavior, which retains at least a portion of its ancestral responsiveness. The emancipation of displacement activities from their original controlling stimuli and their attachment to other types of stimulus control as they become ritualized into displays has been described almost entirely in terms of the communicative functions served. However, there are similarities in response topography dynamics in ritualized and adjunctive behaviors which suggest that the characteristic intensification may have sources other than social display selective pressures. Both behaviors are exaggerated either in movement amplitude or intensity. Both are protracted and acquire rhythmic qualities. Both reveal a "typical intensity" (Morris, 1957): when they occur, their form and intensity resist alterations by stimulus variables that usually produce graded changes in the behavior. There is proof that at least one adjunctive behavior, scheduleinduced polydipsia, is emancipated from its ancestral controlling variables. For example, presession water loading failed to attenuate the polydipsic behavior to a significant degree (Falk, 1969). In contrast, such loading considerably reduces drinking produced by conditions affecting body water status.

Ritualization, the ceremonious exaggeration and persistence of what would appear to be ancillary behaviors under certain environmental conditions, occurs, then, in some situations unrelated to social communicative display. Ritualized behavior may acquire a communicative function in a succeeding phase; but neither its inception as a displacement activity nor its exaggeration into a strong and persistent behavior are necessarily in the service of communication. It is more likely that ritualized or adjunctive behavior is preadapted for elaboration into communicative display functions (Spurway \& Haldane, 1953). Ritualized displays are somewhat maladaptive in that they often make the organism conspicuous to predators as well as to potential mates. Adjunctive behavior too is a considerable investment in time and energy, the utility of which is not readily evident. But undoubtedly selective advantages accrue to species prone to the ritualization of displacement activities under the appropriate environmental conditions. It is probable that this occurs in accordance with Romer's rule: the ritualized or adjunctive behavior maintains adjustment to the current context. By emphasizing an ancillary behavior already possessing some adaptive significance in that situation, the entire context is stabilized and conserved (cf. the diversity-stability rule). Romer's rule, as formulated by Hockett and Ascher (1964) and quoted previously, may now be restated as it applies to the functional significance of adjunctive behavior: The adaptive value of adjunctive behavior consists in its maintenance of the organism's engagement with a problematic, but overall favorable, situation.

What characterizes the "problematic" situations referred to above, and how does adjunctive behavior 
stabilize such situations? Conditions yielding displacement activities-agonistic, mating, incubation, and parental care contexts-were described previously. Their problematic aspect often occurs when the appropriate behaviors are impeded: the antagonist makes the outcome of an agonistic context uncertain, mating encounters run uneven courses fraught with agonistic components, while incubation and parental situations are interrupted by returning mates, group members, or by the approach of potential predators. These conditions involve escape components. But if a territory is to be defended, mating accomplished, or offspring conserved, these situations must be preserved. If escape behavior were to dominate these situations, even momentarily, there would be an appreciable chance that territory, mates, and progeny would be lost. There is danger of such an eventuality in just these types of situations because they are composed of two opposed components (fight vs. flight, etc.) in unstable equilibrium. Adjunctive behaviors or displacement activities have a protective or buffering action in that they effect the continued engagement of the organism with the problematic situation. Timesharing occurs between the problem situation and one or more adjunctive behaviors until resolution of the opposing vectors of the problem situation develops. Given the unstable equilibrium of the generator vectors, the resolution is less likely to be precipitous under circumstances where adjunctive behavior inclusion diversifies the situation. Adjunctive behavior acts as a stabilizing, protective agent, preserving the opposing-vector situation while it develops. This stabilization occurs when ancillary, adaptive behaviors which receive facilitatory stimuli preexistent in the situation become exaggerated and ritualized. Mechanisms apparently have evolved by genetic selection that strengthen adjunctive behavior under the opposing-vector conditions defined by generator situations.

The adaptive significance of adjunctive behavior resides in the several consequences that flow from its incorporation and exaggeration in generator circumstances. (a) Such situations are stabilized by the diversification of the network of behaviors brought about by adjunctive behavior inclusion and persistence. (b) Spatially, adjunctive behavior maintains the organism in the situation by occluding escape behavior, thereby postponing a resolution of the circumstances until the interplay of generator vectors evolves further. This serves to pace the generator-situation behavior, which might otherwise resolve precipitously given the current dangers and constraints. An unstable equilibrium is converted into a stable one, allowing the generator vectors to develop at a measured pace. (c) Finally, adjunctive behaviors are complex responses of con- siderable strength and duration which are preadapted for other potential functions, such as communication, abode construction, and tool use. As Skinner remarked in a passage following his discussion of preadaptation, "behavior may have advantages which have played no role in its selection" (Skinner, 1966).

\section{OF SPECIES AND PATCH FUGACITY}

Some species are highly mobile hunters (many carnivores and herbivorous seed and fruit eaters), exploiting their environmental food sources by moving from place to place. These movements are controlled in a complex manner by the "patchy" availability of food sources, so that as one patch becomes sparce the animal moves to a more favorable one (e.g., Charnov, 1976; MacArthur \& Pianka, 1966). The herbivorous grazers and browsers are less mobile, exploiting relatively small, densely supplied home ranges (McNab, 1963). Still other species move to a favorable place where, with immobile waiting, prey may be ambushed (e.g., bobcats, leopards, and many spiders and snakes). Finally, some animals stay within one locus and capture prey through lure display (e.g., angler fish: Wickler, 1968, p. 124ff).

The mobility of an organism in response to current food availability is a function of the particular species' foraging mode and the food-resource status within the patch of present residence. Species with low mobility as a dominant feature of their foraging mode might be expected to show less escape behavior from a domain of attenuated food acquisition rate. If this is the case, then they should evidence less adjunctive behavior as a result of food schedule manipulation. Adjunctive behavior has been demonstrated in several species; nevertheless, some species may not yield schedule-induced polydipsia (e.g., the golden hamster: Wilson \& Spencer, 1975) or other adjuncts when intermittent-food generator conditions are imposed. It is possible that if adjunctive behavior is, as suggested, a stabilizing activity maintaining the organism's engagement with a situation containing escape components, a minimally fugacious species may not require the diversification of adjunctive behavior to protect a nonoptimal, but feasible, feeding situation. The present evidence on species differences with regard to adjunctive behavior is too fragmentary to enable the extraction of general principles. But it may be of interest to speculate concerning species of intermediate mobility. These include species which are fairly mobile between the long bouts of immobility required for ambush. Finer, less extravagant, stabilizing displacement movements would be expected in such animals for at least two reasons. First, with the rather static ambush 
foraging mode, the escape component generated by feeding intermittence should be greatly diminished. Second, the requirement for stealth would preclude excessive or exaggerated movement. Possibly the small-amplitude tail twitches of felines as they lie in wait for prey to pounce on is a behavioral diversification facilitating the required stasis. Likewise, many snakes vibrate the tail in agonistic situations, a behavior which may occlude escape. Further, in some snakes, tail-tip twitching and coloration have developed to resemble an insect larva. This lure display probably derived from the preadapted ancestral displacement activity. Additional lure displays are described by Alcock (1975, p. 343ff) and Wickler (1968).

It is probable that the contextual conservatism effected by displacement activities which limit the fugacity of ambush feeders evolves into lure displays under the selective pressure of more efficient predation. This may be compared with the enforced limits on fugacity required by human tasks and social interactions which must be done for long periods in one place (e.g., writing an article, assembly line work, crowded parties). These circumstances often induce a common set of adjunctive behaviors: smoking, fluid and food overindulgence, and ritualized verbal displays, all of which function to sustain situational tasks and interactions containing escape components (e.g., Roy, 1960). The adjunctive behaviors block fugacity and keep the organism engaged with the situation-on the job. This, too, can develop into luring in a variety of situations. Consider that exaggerated smoking or gum-chewing movements, or agitated vocal inflections (or fan manipulation in some cultures), function as lures, since they communicate an unstable equilibrium status, and hence possible vulnerability of the displayer.

To return to the initial theme of this section, the very mobile hunters with large prey-search times relative to prey-pursuit times (MacArthur, 1972, p. $61 \mathrm{ff}$ ), with rather low patch-fugacity thresholds, should yield food-schedule-induced adjunctive behavior most readily. The creatures with less mobile modes of foraging may reveal less adjunctive behavior, but it may not be inconsequential. For example, the ambush mode may remain open to lowamplitude adjunctive behavior inclusion as a stabilizer of enforced waiting. Also, they may depend upon high prey abundance, leaving them sensitive to small capture rate decrements.

\section{ADJUNCTIVE BEHAVIOR LAWS}

By way of a summary, four provisional laws are presented with the hope that they can serve to unify the direction of additional research efforts in this area.
(1) Law of adjunct exaggeration. The more constrained a situation (a) spatially, (b) in terms of generator-schedule commodity deficit, or (c) with respect to the competition of scheduled behavior with an escape vector, the greater will be the intensity of the adjunctive behavior.

This law essentially defines the set of conditions governing adjunctive response strengthening. It attempts to state the circumstances increasing the reinforcing properties of specific response patterns and environmental commodities which are involved in the increased probability of behavioral engagement termed "adjunctive behavior." In (a) above, the reference is literally to constrained spatial area. In the usual operant experimental space arrangement, it implies that larger areas should result in somewhat attenuated adjunctive behavior. The immobility contingencies imposed on ambush-mode feeders implies a similar constraint, but there are a more complexly determined set of relevant factors (discussed in the previous section) which limit their adjunctive behavior intensity. In (b) above, most of what is known concerning the relation between commodity deficit and adjunctive behavior has been gathered by food-ration manipulation of body weight, and the inverse relation was discussed briefly in the introduction. In (c), the implication is that as the schedule-determined and escape vectors approach equality, the situation increases in constraint. "Constraint" here should be understood as the necessity of engaging in behavior relevant to two opposing vectors. If one of the vectors departs from the point of equality, it would gain ascendency and the situational constraint would dissipate. Figure 1 shows this constraint relation as it applies to adjunctive behavior generation by schedule induction. The diagram assumes that the conditions described in (a) and (b) have been met, at least to some minimally effective extent. The linear aspect of the function should not be regarded as a necessary feature of the law. The consummatory behavior function (scheduledetermined vector) can be controlled rather precisely by the experimenter and simply reflects the manipulated condition indicated on the $x$-axis. There is little information available on the shape of the escape function. Existing information suggests that it is bitonic (Brown \& Flory, 1972), as one might expect if it were similar to other schedule-induced behaviors (cf. introductory section). However, schedule-induced escape has been explored by allowing animals to escape from the discriminative stimuli associated with schedules, rather than permitting spatial escape. These two kinds of escape may not be equivalent, but only experimentation will tell. The ethological and ecological relations discussed involve mainly spatial escape vectors (encounters containing agonistic components, patch fugacity). The view implied by 


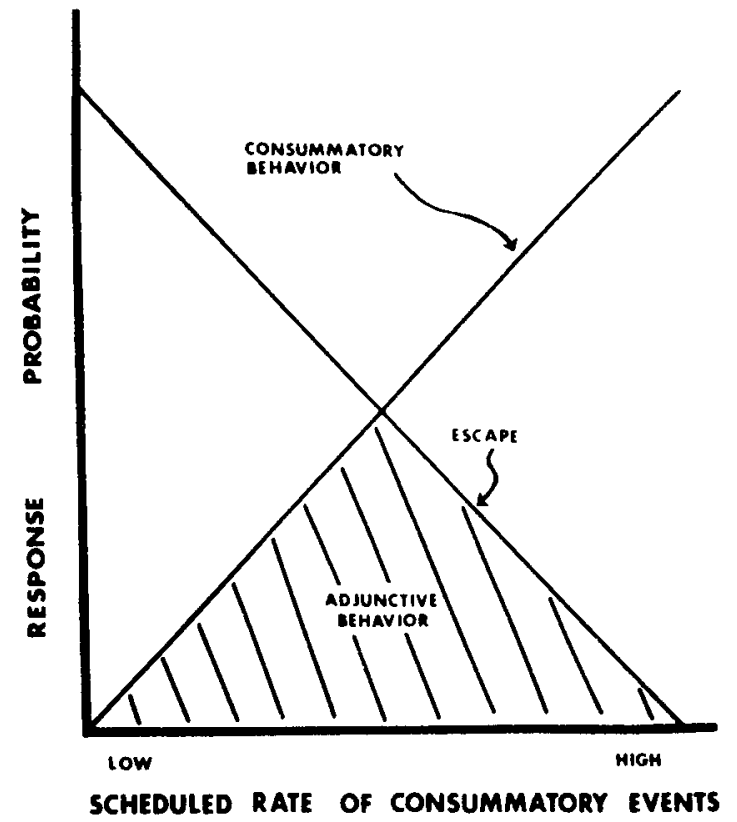

Figure 1. Adjunctive behavior probability as the inverse of the difference between two functions: (1) the rate of generatorschedule consummatory behavior, and (2) the probability of escape behavior.

Figure 1 is that schedule-induced escape has a special status. Its interaction with the consummatory behavior function is responsible for the induction of the other adjunctive behaviors.

(2) Law of adjunct selection. The adjunctive behavior which occurs, other factors remaining equal, is that which receives maximal stimulus facilitation.

Present information on this point is derived mainly from studies on the stimulus facilitation determinants of displacement activities and was discussed in a previous section.

(3) Law of protective inclusion. A situation evoking two competing behavioral vectors of equivalent strength which result in an unstable equilibrium, such that one of the vectors could become eliminated or undergo oscillations of large magnitude, will increase in stability if one or more adjunctive behaviors share the behavioral domain.

(4) Law of minimal intrusion. Other factors remaining equal, the adjunctive behaviors that emerge are those which maintain the organism in closest proximity to the generating situation and occur at times minimally competitive with the behavior primarily determined by the generator schedule.

In accordance with this law, adjunctive behavior typically occurs at postreinforcement times and at points where reinforcement probability is low (Falk, $1969,1971)$. But, as the phrase "other factors remaining equal" intimates, adjunctive behavior can be so intrusive as to interfere with its generating conditions. Often, however, it only exacerbates them. If adjunctive behavior is invasive enough, schedule contingency requirements may not be met, increasing reinforcement intermittency. This could result in a further increase in the adjunct if the intermittence change lies on the rising limb of the bitonic function. A major interference by adjunctive behavior with its generator conditions would, of course, be self-limiting. More often the intrusive or disadvantageous aspect of adjunctive behavior (e.g., excessive smoking, drug taking, loquaciousness) interferes with adaptive behaviors other than those tied to the generating conditions. But excessive and intrusive aspects of adjunctive behavior can be overemphasized. Its major adaptive significance, as the law of protective inclusion states, lies in its stabilizing function.

\section{REFERENCE NOTE}

1. Villarreal, J. Schedule-induced pica. Paper presented at the meeting of the Eastern Psychological Association, Boston, April 1967.

\section{REFERENCES}

Alcock, J. The evolution of the use of tools by feeding animals. Evolution, 1972. 26, 464-473.

Alcock, J. Animal behavior: An evolutionary approach. Sunderland, Mass: Sinauer Associates, 1975.

Allen, J. D., \& Kenshalo, D. R., JR. Schedule-induced drinking as a function of interreinforcement interval in the rhesus monkey. Journal of the Experimental Analysis of Behavior, 1976, 26. 257-267.

Armstrong, E. A. Diversionary display. Part 1. Connotation and terminology. Ibis, 1949, 91, 88-97. (a)

Armstrong. E. A. Diversionary display. Part 2. The nature and origin of distraction display. Ibis, 1949, 91, 179-188. (b)

Armstrong, E. A. The nature and function of displacement activities. Symposia of the Society for Experimental Biology, 1950, 4, 361-384.

Armstrong, E. A. Bird display and behavior (Rev. ed.). New York: Dover, 1965.

Azrin, N. H. Time-out from positive reinforcement. Science, 1961, 133. 382-383.

Azrin. N. H., Hutchinson, R. R., \& Hake, D. F. Extinctioninduced aggression. Journal of the Experimental Analysis of of Behavior, 1966. 9. 191-204.

BLEST, A. D. The evolution of protective displays in the Saturnoidea and Sphingidae (Lepidoptera). Behaviour. 1957, 11, 257-309.

Bock, W. J. Preadaptation and multiple evolutionary pathways. Evolution, 1959. 13, 194.211.

Brown, T. G., \& Flory, R. K. Schedule-induced escape from fixed-interval reinforcement. Joumal of the Experimental Analysis of Behavior, 1972, 17, 395-403.

Charnov, E. L. Optimal foraging, the marginal value theorem. Theoretical Population Biology, 1976, 9. 129-136.

Chillag, D., \& Mendelson, J. Schedule-induced airlicking as a function of body-weight deficit in rats. Physiology and Behavior. 1971. 6. 603-605.

Conen, P. S., \& Looney, T. A. Schedule-induced mirror responding in the pigeon. Journal of the Experimental Analysis of Behavior, 1973, 19, 395-408.

Cowles, R. B. Possible origin of dermal temperature regulation. Evolution, 1958, 12, 347-357.

Dove, L. D. Relation between level of food deprivation and rate of schedule-induced attack. Journal $\overrightarrow{\rho f}$ the Experimental Analysis of Behavior. 1976, 25, 63-68. 
ELton, C. S. The ecology of invasions by animals and plants. London: Methuen, 1958.

FALK, J. L. Production of polydipsia in normal rats by an intermittent food schedule. Science, 1961, 133, 195-196.

FALK, J. L. Schedule-induced polydipsia as a function of fixed interval length. Journal of the Experimental Analysis of Behavior, $1966,9,37-39$.

FALK, J. L. Conditions producing psychogenic polydipsia in animals. Annals of the New York Academy of Sciences, 1969, 157, 569-593.

FALK. J. L. The nature and determinants of adjunctive behavior. Physiology and Behavior, 1971, 6, 577-588.

FALK, J. L., \& SAMSON, H. H. Schedule-induced physical dependence on ethanol. Pharmacological Reviews, 1975, 27, 449-464.

FloRy, R. K. Attack behavior as a function of minimum inter-food interval. Joumal of the Experimental Analysis of Behavior, 1969. 12. 825-828.

FLORY, R. K. The control of schedule-induced polydipsia: Frequency and magnitude of reinforcement. Learning and Motivation, 1971, 2, 215-227.

GeERTz. C. Agricultural involution. Berkeley: University of California Piess, 1963.

Hairston, N. G., Allan, J. D.. Colwell, R. K., Futuyama, D. J., Howell, J., Lubin, M. D., Mathias, J., \& VANDERMEER, J. H. The relationship between species diversity and stability: An experimental approach with protozoa and bacteria. Ecology, 1968, 49, 1091-1101.

Hockett. C. F., \& Ascher. R. The human revolution. American Scientist, 1964, 52, 70-92.

Hutchinson. G. E. Homage to Santa Rosalia or why are there so many kinds of animals? American Naturalist, 1959, 93, $145 \cdot 159$.

Hutchinson, R. R., Azrin, N. H., \& Hunt, G. M. Attack produced by intermittent reinforcement of a concurrent operant response. Joumal of the Experimental Analysis of Behavior, 1968, 11, 489-495.

HuXLEY. J. Introduction. A discussion on ritualization of behaviour in aninals and man. Philosophical Transactions of the Royal Socicty of London, 1966. 251, 249-271.

Kachanoff, R., Leveille, R., Mclelland, J, P.. \& Wayner, M. J. Schedule induced behavior in humans. Physiology and Behavior, 1973, 11. 395-398.

King. G. D. Wheel running in the rat induced by a fixed-time presentation of water. Animal Learning \& Behavior. 1974, 2. 325-328.

LACK. D. Darwin's finches. Cambridge: Cambridge University Press. 1947.

LEIGH, E. G., JR. On the relation between the productivity, biomass, diversity. and stability of a community. Ecology, 1965, 53, 777-783.

Levitsky, D., \& Collier, G. Schedule-induced wheel running. Physiology and Behavior, 1968, 3. 571.573.

MacARThur, R. H. Fluctuations of animal populations, and a measure of community stability. Ecology, 1955, 36, 533-536.

MacArthur, R. H. Geographical ecology: Patterns in the distribution of species. New York: Harper \& Row, 1972.

MacArthur, R. H., \& Pianka, E. R. On optimal use of a patchy environment. American Naturalist, 1966, 100, 603-609.

MAY. R. M. Stability and complexity in model ecosystems. Princeton: Princeton University Press, 1973.

MAYR. E. The emergence of evolutionary novelties. In S. Tax (Ed.), Evolution after Darwin (Vol. 1). Chicago: University of Chicago Press, 1960. Pp. 349-380.

MCFARLAND. D. J. Hunger, thirst and displacement pecking in the Barbary dove. Animal Behaviour, 1965, 13, 293-300.

MCNAB, B. K. Bioenergetics and the determination of home range size. American Naturalist, 1963, 97, 133-140.

Mendelson. J.. \& Chillag, D. Schedule-induced air licking in rats. Physiology and Behavior, 1970, 5, 535-537.

Miller. J. S., \& Gollub. L. R. Adjunctive and operant bolt pecking in the pigeon. Psychological Record, 1974. 24. 203-208.

MorRIs. D. The reproductive behaviour of the zebra finch
(Poephila guttata), with special reference to pseudofemale behaviour and displacement activities. Behaviour, 1954, 6. 271-322.

MorRIs, D. "Typical intensity" and its relation to the problem of ritualization. Behaviour, 1957, 11, 1-12.

Moynihan, M. Some displacement activities of the black-headed gull. Behaviour, 1953, 5. 58-80.

Odum, E. P. Fundamentals of ecology (3rd ed.). Philadelphia: Saunders, 1971.

Rogers, D., \& HubBaRd, S. How the behavior of parasites and predators promotes population stability. In M. B. Usher \& M. H. Williamson (Eds.), Ecological stability. New York: Wiley, 1974. Pp. 99-119.

RoMer. A. S. The vertebrate story. Chicago: University of Chicago Press, 1959.

Rowell, C. H. F. Displacement grooming in the chaffinch. Animal Behaviour, 1961, 9. 38-63.

Roy, D. F. Banana time: Job satisfaction and informal interaction. Human Organization, 1960, 18, 158-168.

Sevenster, P. A causal analysis of a displacement activity (fanning in Gasteroteus aculeatus L.). Behaviour Supplement, $1961,9,1-170$.

SkINNER, B. F. The phylogeny and ontogeny of behavior. Science, $1966,153,1205-1213$.

SKINNER, B. F. The shaping of phylogenic behavior. Journal of the Experimental Analysis of Behavior, 1975, 24, 117-120.

Spurway, H., \& Haldane, J. B. S. The comparative ethology of vertebrate breathing. I. Breathing in newts, with a general survey. Behaviour, 1953, 6, 8-34.

Thompson, D. M. Escape from $S^{D}$ associated with fixed-ratio reinforcement. Journal of the Experimental Analysis of Behavior, 1964, 7, 1-8.

TINBERGEN, N. "Derived" activities; their causation, biological significance, origin, and emancipation during evolution. Quarterly Review of Biology, 1952, 27, 1-32.

TINBERGEN, N. The evolution of signaling devices. In W. Etkin (Ed.). Social behavior and organization among vertebrates. Chicago: University of Chicago Press, 1964. Pp. 206-230.

Tinbergen, N., \& VanIersel, J. J. A. "Displacement reactions" in the three-spined stickleback. Behaviour, 1947, 1, 56-63.

VAnlersel, J. J. A., \& Bol, A. C. A. Preening of two tern species. A study on displacement activities. Behaviour, 1958, 13, 1-88.

Wallace, M., Singer, G., Wayner, M. J., \& Cook, P. Adjunctive behavior in humans during game playing. Physiology and Behavior, 1975, 14, 651-654.

Wallace. M., \& Singer, G. Adjunctive behavior and smoking induced by a maze solving schedule in humans. Physiology and Behavior, 1976, 17, 849-852.

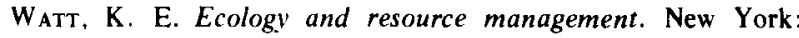
McGraw-Hill, 1968.

W ayner, M. J., Singer, G., Cimino, K., Stein, J., \& Dwoskin, L. Adjunctive behavior induced by different conditions of wheel running. Physiology and Behavior, 1975, 14, 507-510.

WEBBER. M. I. Food web linkage complexity and stability in a model ecosystem. In M. B. Usher \& M. H. Williamson (Eds.), Ecological stability. New York: Wiley, 1974. Pp. 165-176.

WICKLER, W. Mimicry in plants and animals. New York: McGraw-Hill. 1968.

Williamson, K. The distraction behaviour of the Faeroe snipe. Ibis, 1950, 92, 66-74.

Wilson. E. O., \& Bossert, W. H. A primer of population biology. Sunderland, Mass: Sinauer Associates, 1971.

Wilson, S., \& SPEncer, W. B. Schedule-induced polydipsia: Species limitations. Psychological Reports, 1975, 36, 863-866.

WILZ, K. J. Causal and functional analysis of dorsal pricking and nest activity in the courtship of the three-spined stickleback Gasterosteus aculeatus. Animal Behaviour. 1970. 18. $115-124$.

(Received for publication May 6. 1977; revision accepted October $6,1977$. 